vivo) a $120 \mathrm{~K}$ tyrosine kinase with properties very similar to the viral kinase. Although this extends the generality of tyrosine kinase activity as a crucial factor in transformation, it is clear that the small DNA tumour viruses such as SV40 and polyoma lack identifiable kinases. $T$. Benjamin (Harvard University) noted that mutants in the $h r t$ gene region in polyoma virus, which overlaps with the regions encoding the big, middle and small ' $T$ ' antigens, showed decreased levels of histone acetylation compared with wildtype polyoma. All these findings on viral transformation together point to several pathways leading to an ultimate transformation site, and to the ability of different viruses to tap into these different pathways to yield the same overall result.

Returning to the opening question of how many genetic events are required for expression of the transformed phenotype, it seems clear that one is a possibility, but that how many different events can give this apparently single gene change is less clear although one must suspect that it is greater than one. Epidemiologists have argued for multistage models of in situ tumorigenesis, especially for carcinomas where a minimum of between four and six steps is predicted. If one (dominant) or two (recessive) gene level events can give rise to in vitro transformation, then the suspicion must be that the remainder of these steps are either spurious because of assumptions about the data and its relation to tumorigenesis, or are due to evasive steps a transformed cell must take in order to survive various surveillance mechanisms. $\square$

\section{Polarised electrons are going round in circles}

\section{from C. B. Lucas}

SPIN-polarised electrons are of interest to physicists and biologists at both the high and low ends of the particle energy spectrum. Several recent advances have provided new sources of polarised electrons, and should provide new insights in fields such as neutral currents, surface physics and molecular optical activity.

One way of producing spin-polarised electrons is to take a storage ring, fill it with electrons or positrons, and leave for an hour or so. This recipe for polarising electrons by macroscopic means seems contradictory to long-standing arguments that they cannot be so polarised, for example by passage through a SternGerlach magnet. In that case, the splitting of the beam after passing through an inhomogeneous magnetic field is less than the uncertainty in their position as given by Heisenberg's principle.
However the polarisation mechanism is easily understood if one remembers that an electron orbiting in the magnetic field of a storage ring and emitting synchrotron radiation may flip its spin on emitting a photon. The probability of a transition depends on the spin direction relative to the guiding magnetic field, the positrons becoming polarised in the direction of the field, the electrons in the opposite direction. Since the probability of a spinflip emission is typically only $10^{-11}$ that of a normal emission, the polarisation builds up very slowly.

The polarisation effect was first predicted by Ternov, Loskutov and Korovina as early as 1961 . Two years later it was shown that, in a typical storage ring, the transverse polarisation should build up in a period of about an hour to a limiting value of $93.4 \%$.

The first experimental confirmations of theoretical predictions were made on electrons in the storage rings at Novosibirsk and Orsay in 1971. In the former, at an energy of $640 \mathrm{MeV}$, approximately $50 \%$ polarisation was measured after about 100 minutes, compared with a calculated value of $66 \%$ when depolarisation effects were included. Since then, measurements on several of the world's storage rings have been made, confirming these results, and $76 \pm 5 \%$ polarisation has been reported from Stanford. (The latest position was discussed at the Argonne conference on High Energy Physics with Polarised Beams and Polarised Targets (AIP Conference Proceedings No 51, ed. G.H. Thomas, 1979)).

Another example of a circular approach to this problem is the latest experiment to produce polarised electrons by photoionisation with circularly polarised light. This process is known as the Fano effect, after its originator. In this case, polarised electrons are produced by transfer of angular momentum from the photons to the electrons. The first Fanoeffect studies were made in the near ultraviolet with caesium, and were remarkable in producing electrons, almost completely polarised, with wavelengths close to a minimum in the photoionisation cross section.

Measurements in the vacuum ultraviolet require more sophisticated optical techniques. Measured polarisation at wavelengths longer than $140 \mathrm{~nm}$ ranges from a maximum of about $50 \%$ in thallium to, typically, a mere $15 \%$ in lead. Molecules have been even more disappointing, yielding no significant polarisation of the photoelectrons.

Nevertheless measurements have been continued further into the vacuum ultraviolet, at around $100 \mathrm{~nm}$, by a group using the radiation produced by the synchrotron running at $800 \mathrm{MeV}$ at the University of Bonn (J. Phys. B 12, L679; 1979). Preliminary measurements showed that the radiation above and below the plane of the electrons was respectively left and right circularly polarised to at least $80 \%$. When used to ionise xenon, circularly polarised photons produced electrons which were polarised up to $75 \%$, in general agreement with theoretical predictions. Though still not so impressive as the very first measurements in caesium, these results are comparable with the polarisation which could have been obtained if the electrons in the synchrotron had been injected into a storage ring, though the current would then have been many orders of magnitude greater. Hence polarised electrons in a storage ring could be used to produce polarised photoelectrons through the synchrotron radiation! This means that the particle physicist is rapidly losing his interest in the polarised electrons produced by the atomic physicist, since the former has a very intense source of highly polarised energetic electrons (and positrons) accidentally at his disposal; this provides the opportunity to study directly the neutral weak current phenomenon.

The atomic physicist can unfortunately make little use of polarised electrons or positrons at such high energies, and the laws of electron optics prevent a useful well-defined low energy beam from being obtained from one of much higher energy. Of interest, therefore, is the report by a group at the University of Michigan (Phys. Rev. Lett. 43, 1281; 1979) that $1 \mathrm{eV}$ positrons have been produced with at least $20 \%$ polarisation. The authors suggest that the principal use of this source to the physicist will be for the study of polarised low-energy positron diffraction from surfaces. For the biologist, interest in this source is connected with the controversial hypothesis that the origin of biomolecular optical activity might be linked to the polarisation of $\beta$-particles from radionuclides.

Interest in spin-polarised electrons is burgeoning as the number of different mechanisms for their production has increased rapidly in the past decade; previously, only elastic electron scattering by heavy atoms looked at all useful. Production of polarised electrons by the use of lasers has not yet proved to be particularly important. Developments in multiphoton ionisation of alkali atoms look very promising, since the atom can be pumped into a fully polarised excited state and then photoionised with a second circularly polarised laser beam. The electrons are then not only expected to be almost fully polarised, but also to have the least energy spread of any source of electrons, so that interesting experiments with low energy polarised monochromatic electrons should become possible.

C.B. Lucas is a SRC research fellow in the Department of Physics, Royal Holloway College, University of London. 